\title{
Sequence diversity of the mucABD locus in $P$ seudomonas aeruginosa isolates from patients with cystic fibrosis
}

\begin{abstract}
Correspondence
Burkhard Tümmler

tuemmler.burkhard@

mh-hannover.de
\end{abstract}

Received 30 May 2006

Revised 20 July 2006

Accepted 28 July 2006

\author{
Alessandra Bragonzi, ${ }^{1,2}$ Lutz Wiehlmann, ${ }^{3}$ Jens Klockgether, ${ }^{3}$ \\ Nina Cramer, ${ }^{3}$ Dieter Worlitzsch, ${ }^{1}$ Gerd Döring ${ }^{1}$ and Burkhard Tümmler ${ }^{3}$ \\ ${ }^{1}$ Institute of Medical Microbiology and Hygiene, Universitätsklinikum Tübingen, Tübingen, \\ Germany \\ ${ }^{2}$ Institute for Experimental Treatment of Cystic Fibrosis, DIBIT - HS Raffaele, Milano, Italy \\ ${ }^{3}$ Klinische Forschergruppe, OE 6710, Medizinische Hochschule Hannover, Carl-Neuberg-Str. 1, \\ D-30625 Hannover, Germany
}

The mucA gene of the muc operon, which is instrumental in the control of the biosynthesis of the exopolysaccharide alginate, is a hotspot of mutation in Pseudomonas aeruginosa, a micro-organism that chronically colonizes the airways of individuals with cystic fibrosis (CF). The mucA, mucB and mucD genes were sequenced in nine environmental isolates from aquatic habitats, and in $37 P$. aeruginosa strains isolated from 10 patients with CF, at onset or at a late stage of chronic airway colonization, in order to elucidate whether there was any association between mutation and background genotype. The 61 identified single nucleotide polymorphisms (SNPs) segregated into 18 mucABD genotypes. Acquired and de novo stop mucA mutations were present in 14 isolates (38\%) of five mucABD genotypes. $\triangle \mathrm{G} 430$ was the most frequent and recurrent mucA mutation detected in four genotypes. The classification of strains by mucABD genotype was generally concordant with that by genome-wide Spel fragment pattern or multilocus SNP genotypes. The exceptions point to intragenic mosaicism and interclonal recombination as major forces for intraclonal evolution at the mucABD locus.

\section{INTRODUCTION}

The metabolically versatile Pseudomonas aeruginosa is present in soil and aquatic habitats, but it is also an important opportunistic pathogen for humans, animals and plants. Typing of strain collections by single nucleotide polymorphisms (SNPs), DNA fragment length polymorphisms and phenotypic traits indicates that the current $P$. aeruginosa population is in linkage equilibrium, and consists of a network of equivalent genotypes (termed clones), whereby a subset of clones is overrepresented due to epidemic spread (Curran et al., 2004; Morales et al., 2004; Pirnay et al., 2002a, b).

In $P$. aeruginosa, interclonal and intraclonal diversity have been reported to result from the acquisition or loss of genomic islets or islands, genome rearrangements, and recombination or point mutation (reviewed by Tümmler, 2006). Mosaic genes are a further source of genetic diversity. They contain regions with different degrees of relatedness which can be explained by recombination of two distinct alleles as a result of gene transfer events (Hakenbeck, 1998).

Abbreviations: CF, cystic fibrosis; SNP, single nucleotide polymorphism
Evidence for a mosaic gene structure has been drawn from SNP haplotype (Spangenberg et al., 1998; Kiewitz \& Tümmler, 2000) or the detection of cassettes (Spangenberg et al., 1996). Our current knowledge about mosaic genes in $P$. aeruginosa is restricted to the pyoverdine biosynthesis locus (Smith et al., 2006), ampC (PA4110) (Spangenberg et al., 1998), fleP (PA1096) (Arora et al., 2001), fliC (PA1092) (Spangenberg et al., 1998) and oprD (PA0958) (Pirnay et al., 2002b). In the case of oprD, sequence analysis of $55 \mathrm{P}$. aeruginosa isolates, collected over a period of 15 years from various, spatially separated, clinical and environmental habitats, has uncovered a microscale mosaic structure of $o p r D$. All sequences fall into three main groups, which differ by $7-9 \%$ of their nucleotides. Several recombinational exchanges of DNA blocks of 100-300 bp lead to a mosaic gene structure and cause further divergence into subgroups.

Most information about the evolution of intraclonal diversity of $P$. aeruginosa has been obtained from retrospective cross-sectional and longitudinal analyses of isolates recovered from the atypical habitat of the lungs of patients with cystic fibrosis (CF) (Ernst et al., 2003; Kresse et al., 2003; Larbig et al., 2002; Smith et al., 2006). A hallmark of 
the adaptation of $P$. aeruginosa to the atypical niche of the CF lung is the emergence of mucoid alginate-overexpressing morphotypes that are typically associated with stop mutations in the mucA gene (Martin et al., 1993a).

The activation of alginate genes is complex (reviewed by Ramsey \& Wozniak, 2005). The algU gene, which is essential for alginate production, encodes the alternative sigma factor $\sigma^{22}$. MucA is an anti-sigma factor that sequesters $\sigma^{22}$ (Mathee et al., 1997; Schurr et al., 1996), and MucB is a periplasmic negative regulator (Martin et al., 1993b). MucD is a homologue of the Escherichia coli periplasmic serine protease HtrA, and is apparently both a chaperone and a regulator of alginate production (Wood \& Ohman, 2006).

This study reports on the sequence diversity of the mucABD operon in nine environmental and 37 CF $P$. aeruginosa isolates. The association between mucABD SNP haplotype and chromosomal genotype has been inferred from SpeI restriction fragment patterns (Römling et al., 1994) and multilocus SNP genotypes (Morales et al., 2004). The CF strains were isolated from 10 patients at onset and at a chronic late stage of airway colonization. In living patients, the late isolates were collected after 13-15 years of continuous chronic airway colonization, and in deceased patients, the last isolates prior to death were studied. Sequence analysis of sequential isolates has so far only been performed on strains from a young $\mathrm{CF}$ patient who acquired $P$. aeruginosa during infancy, and who was monitored for a period of 8 years thereafter (Spencer et al., 2003; Smith et al., 2006).

Since mucA mutations are common in CF isolates (Martin et al., 1993a, b), the sequence analysis provided the opportunity to investigate the diversity of the genetic background in a mutation hotspot in the $P$. aeruginosa genome. The comparative study of sequential isolates allowed us to differentiate between acquired and de novo mutations. Sequence and genotype analysis provided direct evidence for recurrent mucA mutations and the generation of mosaic muc genes in $P$. aeruginosa that persists in CF lungs.

\section{METHODS}

CF patients, bacterial strains and growth conditions. $P$. aeruginosa strains were isolated from 10 pancreatic-insufficient $\mathrm{CF}$ patients attending the CF clinic of the Medizinische Hochschule Hannover. Thirty-seven $P$. aeruginosa strains were analysed from sputa or throat swabs from the $10 \mathrm{CF}$ patients (Table 2). Twenty $P$. aeruginosa strains were collected at the onset of chronic colonization. From the five living patients (patients 1-5), eight additional strains were collected 13-15 years later. From the five deceased patients (patients 6-10), the last isolate collected prior to death 5-14 years after acquisition of $P$. aeruginosa was analysed. Strains were stored at $-70{ }^{\circ} \mathrm{C}$, and were not subcultured. In addition, the completely sequenced strains $P$. aeruginosa PAO1 and PA14, and nine environmental isolates from aquatic habitats (SG17M, PT2, PT6, PT12, PT20, PT22 and DSM939), mushroom (ATCC 33818) and a fuel tank (ATCC 33988) were studied.

Genotyping by PFGE. Samples were analysed as described by Römling et al. (1994), with minor modifications. P. aeruginosa strains were grown overnight at $37^{\circ} \mathrm{C}$ in $\mathrm{LB}$ medium, centrifuged for $10 \mathrm{~min}$ at $1500 \mathrm{~g}$, and suspended in $75 \mathrm{mM} \mathrm{NaCl}, 25 \mathrm{mM}$

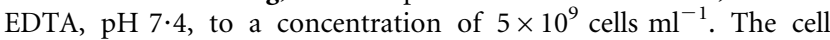
suspension was mixed 1:1 with $2 \%(\mathrm{w} / \mathrm{v})$ low-melting-point agarose to prepare the agarose plugs. The embedded cells were digested for $48 \mathrm{~h}$ at $56^{\circ} \mathrm{C}$ with proteinase $\mathrm{K}\left(1 \mathrm{mg} \mathrm{ml}^{-1}\right.$ in $0.5 \mathrm{M}$ EDTA, $\mathrm{pH} 9 \cdot 5,1 \%, \mathrm{v} / \mathrm{v}, \mathrm{N}$-lauryl sarcosine). Agarose plugs were equilibrated and stored in $10 \mathrm{mM}$ EDTA, $10 \mathrm{mM}$ Tris/ $\mathrm{HCl}, \mathrm{pH} 7 \cdot 4$, at $4{ }^{\circ} \mathrm{C}$ until used. One-third to one-half of a plug was equilibrated in SpeI buffer $(50 \mathrm{mM} \mathrm{NaCl}, 6 \mathrm{mM}$ Tris/HCl, pH 7·5, $10 \mathrm{mM} \mathrm{MgCl}$ ). Digestion was performed overnight at $37^{\circ} \mathrm{C}$ in $90 \mu$ l enzyme buffer with $4 \mathrm{U}$ SpeI, $0 \cdot 1 \mathrm{mg} \mathrm{BSA} \mathrm{ml}{ }^{-1}$ and $5 \mathrm{mM}$ DTT. Separation of DNA fragments was performed by PFGE in a CHEF-DR III apparatus (Bio-Rad), using a $1.5 \%(\mathrm{w} / \mathrm{v})$ agarose gel, $0.5 \times$ Tris/borate/ EDTA buffer, and a linear ramping of $8-50 \mathrm{~s}$ for $24 \mathrm{~h}, 12-25 \mathrm{~s}$ for $22 \mathrm{~h}$, and $1-14 \mathrm{~s}$ for $14 \mathrm{~h}$. DNA fragments were visualized by ethidium bromide staining. Genotypes were defined from the evaluation of SpeI fragment patterns, as described by Römling et al. (1994).

Multilocus SNP genotyping. Boiled colonies of each strain were subjected to PCR with appropriate primers to amplify DNA fragments containing the SNP, as described by Morales et al. (2004). Amplified DNA was digested with the restriction enzyme that discriminated each SNP (Table 1), and the fragments generated were analysed by agarose gel electrophoresis and ethidium bromide staining. To detect the SNP in the oprL gene, an artificial restriction site was created in the direct primer, and 'mismatch PCR' was performed. In brief, the primer was designed so that its $3^{\prime}$ end was 1 bp upstream from the SNP, and it contained a mismatch close to the $3^{\prime}$ end, which generated a restriction site for BstUI when the sequence contained a cytosine at the site of the SNP, but not when the base was a thymidine. Therefore, the presence or absence of the BstUI site allowed analysis of the SNP.

Analysis of flic, exos and exoU. P. aeruginosa strains contain one of two variants of the fliC gene ( $\mathrm{a}$ or b type), which encode flagellins of different molecular mass (Spangenberg et al., 1998), and one or no copies of exoS or exoU, which encode type III secretion effector proteins. Most strains encode either exoS or exoU (Feltman et al., 2001). Therefore, the presence of exoS and exoU and the fliC type were established for each strain by PCR.

Sequence analysis at the muc locus. One loop of a single $P$. aeruginosa colony, grown on blood agar, was processed for DNA extraction using a commercial DNA isolation kit (Qiagen), according to the instructions of the manufacturer. PCR amplification of the entire $m u c A, m u c B$ and $m u c D$ genes was carried out using Taq DNA polymerase (Qiagen). The following primers (MWG-Biotech) were used: $m u c A, 5^{\prime}$-CTC GTG AAG CAA TCG ACA AA-3' and 5'-AAA AGC AAC AGG GAG GTG GT-3'; mucB, 5'-ATC CGC CGT CAG TGG TAC AG-3' and 5'-CGA GCA GGA CGA GCA GGT AC-3'; and $m u c D, 5^{\prime}$-GTC CGA TTC GGC CTG AGT CT- $3^{\prime}$ and $5^{\prime}$-ACG CAG GTA ACG GAT TGA CG-3'. The amplified DNA samples were sequenced by standard automated DNA sequence technology employing the primers described above and the following additional internal primers for $m u c B$ and $m u c D$ : $m u c B$ int1, $5^{\prime}$-CAG TGG TCC TTG CGG TGA CT- $3^{\prime}$ and $m u c B$ int $2,5^{\prime}$-TTC AGC AGC AGC GAC TTC AA-3', and $m u c D$ int $1,5^{\prime}$-GAT CAA CCC GGG TAA CTC CG$3^{\prime}$ and $m u c D$ int $2,5^{\prime}$-AGA TCT GCG AGT TGA TGC CG-3'. The sequence results were compared with the strain $\mathrm{PAO} 1$ sequence (www.pseudomonas.com) by the BLAST program at the NCBI database (www.ncbi.nlm.nih.gov/blast/), in order to determine the occurrence of sequence variants within the $m u c$ gene cluster.

The relatedness of strains by mucABD or multilocus genotype was calculated by parsimony analysis with the program PARS of the phylogenetic software package PHYLIP version 3.6 (alpha 3) (Felsenstein, 2002). 
Table 1. Multilocus SNP genotyping of $P$. aeruginosa

The sequences of the P. aeruginosa strains PA103 (exoU), PAK (fliC a type) and PAO1 (all other genes) were taken as reference.

\begin{tabular}{|llcl|}
\hline Locus & Restriction enzyme & $\begin{array}{c}\text { SNP or presence } \\
\text { of gene (see Fig. 1) }\end{array}$ & Designation \\
\hline oriC & AlwNI & T267C & I \\
oprL & BstUI & T396C & II \\
fliC a & MspA1I & A672T & III \\
alkB2 & MspA1I & G471A & IV \\
citS & AciI & A533G & V \\
& MnlI & G896C & VI \\
oprI & AciI & T195C & VII \\
ampC & MspI & G789A & VIII \\
& BstUI & C1060T & IX \\
& HinfI & G1150A & X \\
& AlwNI & G1166C & XI \\
& AgeI & T1585C & XII \\
fliC & BsrI & C1639A & XIII \\
fliC & & a type & XIV \\
exoS & & b type & XV \\
exoU & & & XVI \\
& & & XVII \\
\hline
\end{tabular}

Alginate determination. For the quantitative determination of alginate production by $P$. aeruginosa patient isolates, the carbazole assay was used (Lam et al., 1980). Briefly, P. aeruginosa was first cultured aerobically in trypticase soy broth (Oxoid) overnight at $37^{\circ} \mathrm{C}$, then plated on 'Pseudomonas isolation agar' containing $1 \%$ glycerol, $\mathrm{v} / \mathrm{v}$, as a carbon source, and incubated for $24 \mathrm{~h}$ at $37^{\circ} \mathrm{C}$, followed by determination of alginate production. For uronic acid detection, growth from three different plates was pooled. The uronic acid content was normalized by measuring the protein content (Bio-Rad).

\section{RESULTS}

\section{Sequence diversity of the mucABD locus}

Sequencing of the mucA, mucB and mucD genes in 37 sequential $P$. aeruginosa strains from $10 \mathrm{CF}$ patients (Table 2) and in nine environmental isolates revealed 61 SNPs (Table 3 ) corresponding to $2 \%$ sequence diversity at this locus, which is significantly higher than the mean sequence diversity of $0.5 \%$ in the $P$. aeruginosa genome (Spencer et al., 2003). The overrepresentation of transitions $(83 \%)$ and synonymous substitutions $(80 \%)$ is similar to that found for evolutionarily neutral SNPs in other loci (Kiewitz \& Tümmler, 2000) or in the bulk genome (Spencer et al., 2003). The $61 \mathrm{SNPs}$ segregated into 18 mucABD genotypes, one of which split into two variants (Table 2, Figs 1 and 2). The largest divergence of the mucABD sequence was observed between the completely sequenced reference strains PAO1 and PA14, the latter represented in our panel by one environmental and five CF isolates (Fig. 1).

Of the 14 non-synonymous nucleotide substitutions, the two common valine-isoleucine polymorphisms I137V and
V441I and the sequence variant Q225E in $m u c D$ were found in more than one SNP genotype. All other amino acid substitutions were specific for a single SNP genotype, including the three non-conservative substitutions S113F in $m u c B$ and $W 171 \mathrm{R}$ and $\mathrm{S} 363 \mathrm{~N}$ in $m u c D$. A representation of Dayhoff's mutation odds matrix (Dayhoff et al., 1978) by projecting the matrix using multidimensional scaling (French \& Robson, 1983; Taylor, 1986) indicated that these three amino acid replacements at least were not neutral; however, it is not yet known whether any substitution modulates the function of the gene product.

\section{Comparison of the mucABD single locus genotype with the genome-wide SNP and Spel fragment genotype: evidence for intragenic mosaicism and interclonal recombination}

The sequential isolates from individual patients belonged either to one clone (three patients) or to two clones (seven patients) (Table 2). Four clones (A, C, D and L; Table 2) were detected in more than one patient. Environmental and CF isolates of the abundant clones C (Dinesh et al., 2003) and D (strain PA14; Liberati et al., 2006) were identical in their mucABD sequences (Fig. 1).

The classification by mucABD genotype corresponded for most strains with that by genome-wide multilocus SNP and anonymous macrorestriction fragment genotypes (Table 2, Fig. 1). Differences were taken as evidence for intra- or interclonal exchange of sequence at the muc locus. First, unrelated clones could share the same mucABD sequence. One set was represented by clones I and L, the other set by 
Table 2. $P$. aeruginosa sequential isolates from CF patients

Strain types: the genome-wide multilocus SNP genotype is indicated by upper-case type, the mucABD genotype by lower-case type, and the SpeI genomic fragment pattern genotype by upper-case Greek type. Strains of PFGE genotype $\Gamma$ belong to the abundant clone $C$ (Dinesh et al., 2003), and strains of genotype $\Delta$ belong to clone PA14 (Liberati et al., 2006).

\begin{tabular}{|c|c|c|c|c|c|c|c|c|}
\hline Patient & $\begin{array}{c}\text { Age at onset } \\
\text { of colonization (years) }\end{array}$ & Strain & $\begin{array}{l}\text { Colonization } \\
\text { time (years) }\end{array}$ & $\begin{array}{l}\text { Alginate }[\mu \mathrm{g} \text { uronic } \\
\left.\operatorname{acid}(\mu \mathrm{g} \text { protein })^{-1}\right]^{*}\end{array}$ & $\begin{array}{c}\text { Multilocus SNP } \\
\text { genotype }\end{array}$ & $\begin{array}{l}\text { mucABD } \\
\text { genotype }\end{array}$ & $\begin{array}{l}\text { mucA stop } \\
\text { mutation }\end{array}$ & $\begin{array}{c}\text { PFGE } \\
\text { genotype }\end{array}$ \\
\hline \multirow[t]{4}{*}{ Patient 1} & $7 \cdot 4$ & RP1 & 0 & $0 \cdot 02$ & A & $\mathrm{a}$ & No & A \\
\hline & & RP2 & 0 & $0 \cdot 02$ & $\mathrm{~A}$ & $\mathrm{a}$ & No & A \\
\hline & & RP73 & $17 \cdot 5$ & $0 \cdot 01$ & $\mathrm{~B}$ & $\mathrm{~b}$ & No & B \\
\hline & & RP74 & $17 \cdot 5$ & $0 \cdot 01$ & $\mathrm{~B}$ & $\mathrm{~b}$ & No & B \\
\hline \multirow[t]{4}{*}{ Patient 2} & $12 \cdot 5$ & SG1 & 0 & $0 \cdot 01$ & A & $\mathrm{a}$ & No & $\mathrm{A}$ \\
\hline & & SG2 & $2 \cdot 1$ & $0 \cdot 06$ & $\mathrm{C}$ & c & $\Delta \mathrm{G} 430$ & $\Gamma$ \\
\hline & & SG57 & $15 \cdot 8$ & $0 \cdot 01$ & A & $\mathrm{a}$ & No & A \\
\hline & & SG58 & $15 \cdot 8$ & $0 \cdot 01$ & $\mathrm{~A}$ & a & No & A \\
\hline \multirow[t]{3}{*}{ Patient 3} & $10 \cdot 6$ & NN1 & 0 & $0 \cdot 01$ & $\mathrm{C}$ & c & No & $\Gamma$ \\
\hline & & NN2 & 0 & $0 \cdot 01$ & $\mathrm{C}$ & c & No & $\Gamma$ \\
\hline & & NN84 & $16 \cdot 3$ & 0.03 & $\mathrm{D}$ & $\mathrm{d}$ & $\mathrm{C} 352 \mathrm{~T}$ & $\Delta 1$ \\
\hline \multirow[t]{4}{*}{ Patient 4} & $13 \cdot 8$ & BT1 & 0 & $0 \cdot 03$ & $\mathrm{D}$ & d & T412C C505T & $\Delta 2$ \\
\hline & & BT2 & 0 & $12 \cdot 1$ & $\mathrm{D}$ & $\mathrm{d}$ & C505T & $\Delta 2$ \\
\hline & & BT72 & $15 \cdot 8$ & $1 \cdot 2$ & $\mathrm{D}$ & d & $\mathrm{C} 505 \mathrm{~T}$ & $\Delta 2$ \\
\hline & & BT73 & $16 \cdot 3$ & $6 \cdot 1$ & $\mathrm{D}$ & $\mathrm{d}$ & $\mathrm{C} 505 \mathrm{~T}$ & $\Delta 2$ \\
\hline \multirow[t]{3}{*}{ Patient 5} & $19 \cdot 5$ & KB1 & 0 & $0 \cdot 01$ & $\mathrm{E}$ & el & No & $\mathrm{E}$ \\
\hline & & $\mathrm{KB} 2$ & $0 \cdot 9$ & $0 \cdot 02$ & $\mathrm{E}$ & e2 & No & E \\
\hline & & KB66 & $15 \cdot 8$ & $0 \cdot 02$ & $\mathrm{~F}$ & $\mathrm{f}$ & G474A $\Delta \mathrm{C} 484$ & $\mathrm{Z}$ \\
\hline \multirow[t]{4}{*}{ Patient 6} & $3 \cdot 9$ & $\mathrm{AA} 1$ & 0 & $0 \cdot 01$ & G & g & No & $\mathrm{H}$ \\
\hline & & AA2 & $0 \cdot 5$ & $0 \cdot 03$ & $\mathrm{H}$ & $\mathrm{h}$ & No & $\Theta$ \\
\hline & & AA43 & $7 \cdot 5$ & $1 \cdot 0$ & $\mathrm{H}$ & $\mathrm{h}$ & $\Delta \mathrm{A} 358$ & $\Theta$ \\
\hline & & AA44 & $7 \cdot 5$ & $0 \cdot 04$ & $\mathrm{H}$ & $\mathrm{h}$ & $\Delta \mathrm{G} 430$ & $\Theta$ \\
\hline \multirow[t]{4}{*}{ Patient 7} & $7 \cdot 5$ & TR1 & 0 & $0 \cdot 01$ & I & $\mathrm{i}$ & $\Delta \mathrm{G} 430$ & I \\
\hline & & TR2 & 0 & $0 \cdot 01$ & I & $\mathrm{i}$ & $\Delta \mathrm{G} 430$ & I \\
\hline & & TR66 & $12 \cdot 8$ & $0 \cdot 02$ & I & $\mathrm{i}$ & No & I \\
\hline & & TR67 & $13 \cdot 5$ & $3 \cdot 9$ & I & $\mathrm{i}$ & $\Delta \mathrm{G} 430$ & I \\
\hline \multirow[t]{4}{*}{ Patient 8} & $13 \cdot 2$ & MF1 & 0 & $0 \cdot 01$ & $\mathrm{~J}$ & j & No & $\mathrm{K}$ \\
\hline & & MF2 & 0 & $0 \cdot 01$ & $\mathrm{~J}$ & j & No & $\mathrm{K}$ \\
\hline & & MF51 & $10 \cdot 1$ & $0 \cdot 03$ & $\mathrm{~J}$ & j & No & $\mathrm{K}$ \\
\hline & & MF52 & $10 \cdot 1$ & $0 \cdot 01$ & $\mathrm{~K}$ & $\mathrm{k}$ & No & $\Lambda$ \\
\hline \multirow[t]{4}{*}{ Patient 9} & $17 \cdot 0$ & KK1 & 0 & $0 \cdot 01$ & $\mathrm{~L}$ & $\mathrm{i}$ & No & M1 \\
\hline & & KK2 & 0 & $0 \cdot 01$ & $\mathrm{~L}$ & $\mathrm{i}$ & $\Delta \mathrm{GT} 400 \Delta \mathrm{G} 430$ & M1 \\
\hline & & KK71 & $12 \cdot 6$ & $0 \cdot 01$ & $\mathrm{~L}$ & $\mathrm{i}$ & No & M1 \\
\hline & & KK72 & $12 \cdot 6$ & $0 \cdot 01$ & $\mathrm{~L}$ & $\mathrm{i}$ & No & M1 \\
\hline \multirow[t]{3}{*}{ Patient 10} & $8 \cdot 1$ & BST1 & 0 & $0 \cdot 02$ & M & $\mathrm{m}$ & No & $\mathrm{N}$ \\
\hline & & BST2 & $1 \cdot 4$ & $0 \cdot 02$ & $\mathrm{~L}$ & $\mathrm{i}$ & No & M2 \\
\hline & & BST44 & $4 \cdot 5$ & $0 \cdot 01$ & $\mathrm{~L}$ & $\mathrm{i}$ & $\Delta \mathrm{G} 430$ & M2 \\
\hline
\end{tabular}

${ }^{\star}$ The alginate contents of the PAO1 strain and of its isogenic PDO300 mutant (Mathee et al., 1999) harbouring the mucA stop mutation $\Delta \mathrm{G} 430$ were $0 \cdot 01$ and $2 \cdot 7 \mu \mathrm{g}$ uronic acid $(\mu \mathrm{g} \text { protein })^{-1}$, respectively.

clone $\mathrm{H}$ and the environmental isolate PT6. In other words, the same mucABD genotype was observed in different genomic backgrounds. Second, the clones $B$ and $H$ represented by strains RP73 and RP74, and AA2, AA43, AA44 and PT6, respectively, shared an identical SNP pattern in $m u c A, m u c B$ and the $5^{\prime}$ portion of $m u c D$, but differed in their SNP pattern $3^{\prime}$ of SNP32 (Fig. 1), indicating that these two mucABD genotypes emerged by intragenic recombination.
Evidence for intragenic recombination at the muc locus was derived not only from interclonal, but also from intraclonal sequence comparison. The first case was the strains KB1 and KB2 which were retrieved from the same patient's lungs within the first year of colonization with $P$. aeruginosa. The two isolates were perfectly matched in their genome-wide SNP and SpeI fragment genotypes, but differed in their mucA SNP genotype, with the mucBD genotypes being identical (Fig. 1). Since it is extremely unlikely that three 
Table 3. SNPs in the mucABD locus

\begin{tabular}{|c|c|c|}
\hline SNP no.* & Sequence variant $\dagger$ & Frequency $\ddagger$ \\
\hline \multicolumn{3}{|l|}{$m u c A$} \\
\hline 1 & T126C, s & $8(17)$ \\
\hline 2 & T133C, s & $10(21)$ \\
\hline 3 & C156T, s & $7(15)$ \\
\hline 4 & C188G, A-G & $1(2)$ \\
\hline 5 & T198G, s & $8(17)$ \\
\hline 6 & G237C, E-D & $1(2)$ \\
\hline 7 & A243G, s & $7(15)$ \\
\hline 8 & G267A, s & $1(2)$ \\
\hline 9 & $\mathrm{~A} 342 \mathrm{G}, \mathrm{s}$ & $47(98)$ \\
\hline 10 & A358T, T-S & $1(2)$ \\
\hline 11 & $\mathrm{C} 360 \mathrm{~A}, \mathrm{~s}$ & $1(2)$ \\
\hline 12 & T381G, s & $7(15)$ \\
\hline \multicolumn{3}{|l|}{$m u c B$} \\
\hline 13 & T90C, s & $1(2)$ \\
\hline 14 & T195C, s & $8(17)$ \\
\hline 15 & C197T, A-V & $3(6)$ \\
\hline 16 & $\mathrm{C} 255 \mathrm{~T}, \mathrm{~s}$ & $7(15)$ \\
\hline 17 & C336T, s & $8(17)$ \\
\hline 18 & C338T, S-F & $10(21)$ \\
\hline 19 & G342A, s & $9(19)$ \\
\hline 20 & C390T, A-V & $1(2)$ \\
\hline 21 & T411C, s & $8(17)$ \\
\hline 22 & T420C, s & $8(17)$ \\
\hline 23 & G486A, s & $10(21)$ \\
\hline 24 & G586T, A-S & $6(13)$ \\
\hline 25 & A631G, T-A & $8(17)$ \\
\hline 26 & A651G, s & $8(17)$ \\
\hline \multicolumn{3}{|l|}{$m u c D$} \\
\hline 27 & G183T, s & $10(21)$ \\
\hline 28 & G231A, s & $9(19)$ \\
\hline 29 & G252A, s & $1(2)$ \\
\hline 30 & G267C, s & $46(96)$ \\
\hline 31 & T294C, s & $7(15)$ \\
\hline 32 & $\mathrm{C} 345 \mathrm{~T}, \mathrm{~s}$ & $1(2)$ \\
\hline 33 & T406C, s & $18(38)$ \\
\hline 34 & A409G, I-V & $18(38)$ \\
\hline 35 & G417A, s & $5(10)$ \\
\hline 36 & T511C, W-R & $2(4)$ \\
\hline 37 & T582G/C, s/s & $32(67) / 6(13)$ \\
\hline 38 & T645C, s & $8(17)$ \\
\hline 39 & T657C, s & $1(2)$ \\
\hline 40 & C673G, Q-E & $7(15)$ \\
\hline 41 & C732T, s & $3(6)$ \\
\hline 42 & T747C, s & $8(17)$ \\
\hline 43 & $\mathrm{C} 774 \mathrm{~T}, \mathrm{~s}$ & $1(2)$ \\
\hline 44 & T804C, s & $13(27)$ \\
\hline 45 & C819T, s & $12(25)$ \\
\hline 46 & G876A, s & $2(4)$ \\
\hline 47 & A897G, s & $13(27)$ \\
\hline 48 & C912T, s & $13(27)$ \\
\hline 49 & T927C, s & $15(31)$ \\
\hline 50 & G1017A, s & $4(8)$ \\
\hline 51 & T1047C, s & $5(10)$ \\
\hline
\end{tabular}

Table 3. cont.

\begin{tabular}{|ccc|}
\hline SNP no.* & Sequence variant $\dagger$ & Frequency $\ddagger$ \\
\hline 52 & C1069T, s & $9(19)$ \\
53 & C1088A, S-N & $5(10)$ \\
54 & G1135A, A-T & $1(2)$ \\
55 & C1170T, s & $1(2)$ \\
56 & C1290T, s & $2(4)$ \\
57 & G1302A, s & $2(4)$ \\
58 & G1321A, V-I & $9(19)$ \\
59 & G1377A, s & $9(19)$ \\
60 & A1389G, s & $9(19)$ \\
61 & T1404C, s & $9(19)$ \\
\end{tabular}

${ }^{\star} \mathrm{SNP}$ no. used in Fig. 1.

$\dagger$ The sequence of the $P$. aeruginosa PAO1 strain was taken as a reference.

$¥$ The frequency of strains carrying the non-PAO variant is given as an absolute number and as a percentage of the investigated strain panel (in parentheses). The frequencies are only rough estimates of the frequency of the SNPs in the P. aeruginosa population, because in numerous cases sequential isolates of the same clone were sequenced (see Fig. 1, Table 2).

successive mutations at common SNP positions occurred de novo, one strain must have incorporated its $m u c A$ gene from another $P$. aeruginosa clone. The second case was the clone $G$ strains AA1 and PT20 which had been isolated in 1985 and 1992, at a distance of $300 \mathrm{~km}$ from one another, from CF airways and a sanitary facility, respectively. The two strains were identical in their genomic SNP genotype and most muc sequences, but differed in the outermost SNPs at the $5^{\prime}$ end of $m u c A$ and the $3^{\prime}$ end of $m u c D$. The genomically related mushroom isolate ATCC 33818 shared the mucA sequence with strain PT20 and the $m u c D$ sequence with strain AA1 (Fig. 1). Hence, two recombinations in the muc locus can explain the emergence of three related mosaic muc genotypes.

\section{mucA stop mutations in CF isolates: frequent recurrent mutations}

Unequivocal loss-of-function stop mutations were identified in the $m u c A$ gene of 14 of our 37 CF isolates (38\%), but in none of the nine environmental isolates that were sequenced in the mucABD genes. No loss-of-function mutations were detected in $m u c B$ or $m u c D$. mucA stop mutations were associated with five mucABD genotypes (Fig. 2), and were observed with similar frequency in strains recovered from both healthier and more severely ill patients (Table 2) (chi-square test: $P=1$ ). Two C-to-T transitions, numerous single nucleotide deletions and two double mutations were observed (Table 2). Four of the six different mutations were located in stretches of thermodynamically stable double strands with $>80 \%$ GC content. 


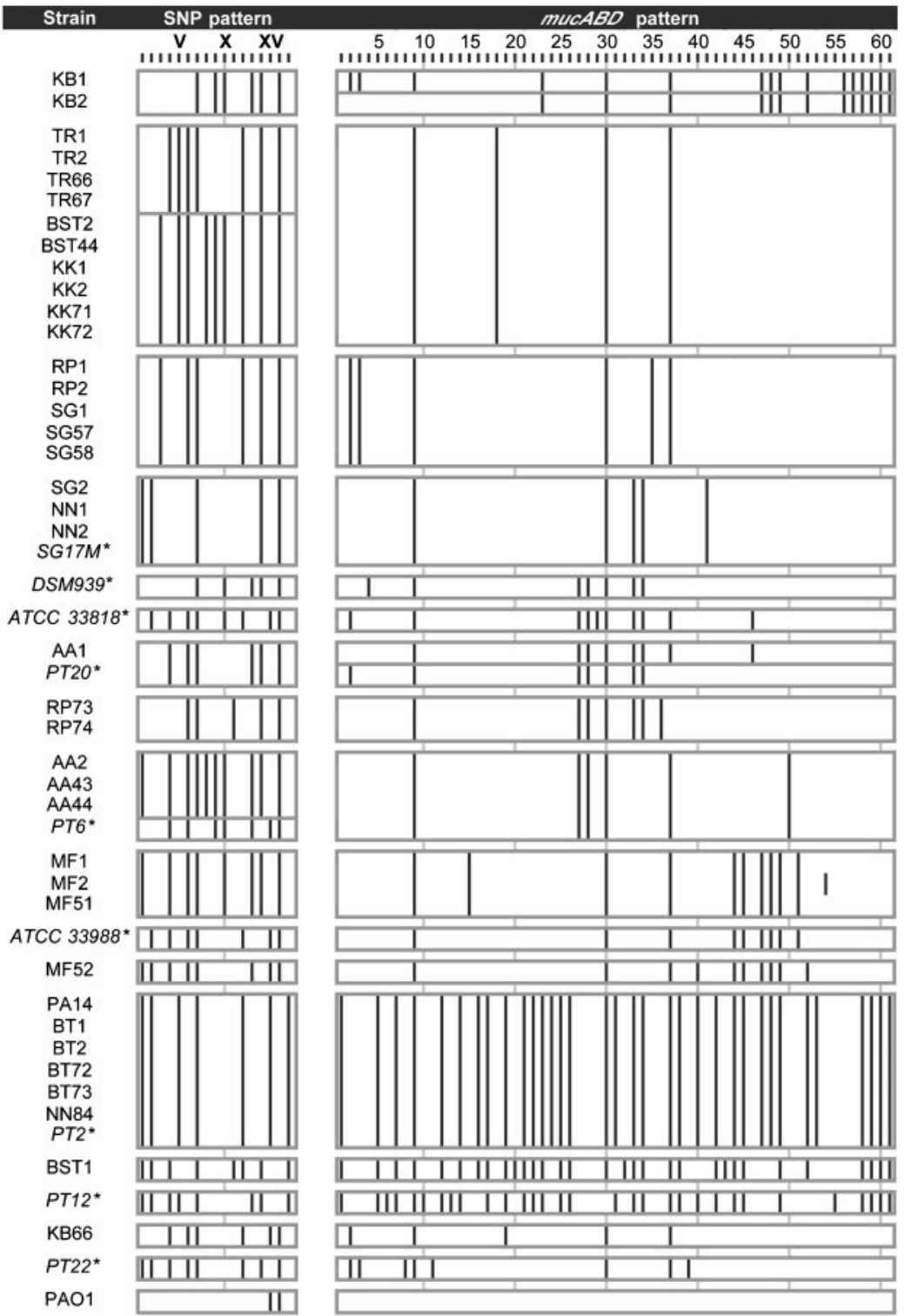

Fig. 1. Multilocus genome-wide SNP genotypes (roman numerals; see Table 1) and mucABD single-locus SNP genotypes (arabic numerals; see Table 3 ) of 37 sequential $P$. aeruginosa isolates from 10 individuals with $\mathrm{CF}$, and nine environmental isolates. The sequenced $P$. aeruginosa strain PAO1 was taken as a reference. Environmental strains are designated by italic type and an asterisk.

mucA mutations have been reported to arise in $P$. aeruginosa during the stage of chronic colonization, when the strains become mucoid (Spencer et al., 2003). However, in our cohort, initial isolates from the first $P$. aeruginosa-positive sputum from patients 4,7 and 9 carried mucA mutations (Table 2). These three patients became positive for $P$. aeruginosa after discharge from a stay at a CF ward, or after return from a $\mathrm{CF}$ rehabilitation centre, indicating that their $m u c A$ mutations were acquired with the initially colonizing strain from an external CF-related source. This suspicion of nosocomial acquisition is supported by the phenotypes of the initial isolates that are typically observed in samples retrieved from chronically colonized CF lungs. The strains from these three patients were mucoid and/or resistant to antipseudomonal agents.

In patients $2,3,5,6$ and $10, m u c A$ stop mutations were detected in just one isolate per patient, which strongly suggests that the mutations had emerged de novo in the CF lung habitat. Half of all the mutations consisted of a deletion of a $G$ in a string of five $G$ residues located at positions $426-430$ in a GC-rich stretch of the mucA gene. This $\Delta \mathrm{G} 430$ deletion occurred in four mucABD haplotypes (Table 2, Fig. 2), which is proof of recurrent mutations on diverse genetic backgrounds.

Recent sequence analyses of the mucA gene in large collections of CF isolates from North America (Spencer et al., 2003; Yoon et al., 2006), Australia (Anthony et al., 2002) and Europe (this study) have identified insertions or deletions in 18 strains, and 21 different stop mutations in 92 strains. More than $80 \%$ of the frameshift or nonsense mutations that give rise to a premature stop signal were clustered within the $100 \mathrm{bp}$ stretch from nt 340-439 of the 585 bp mucA gene (Fig. 3). The most frequent $\Delta \mathrm{G} 430$ mutation accounted for $40 \%$ of all stop mutations. The 


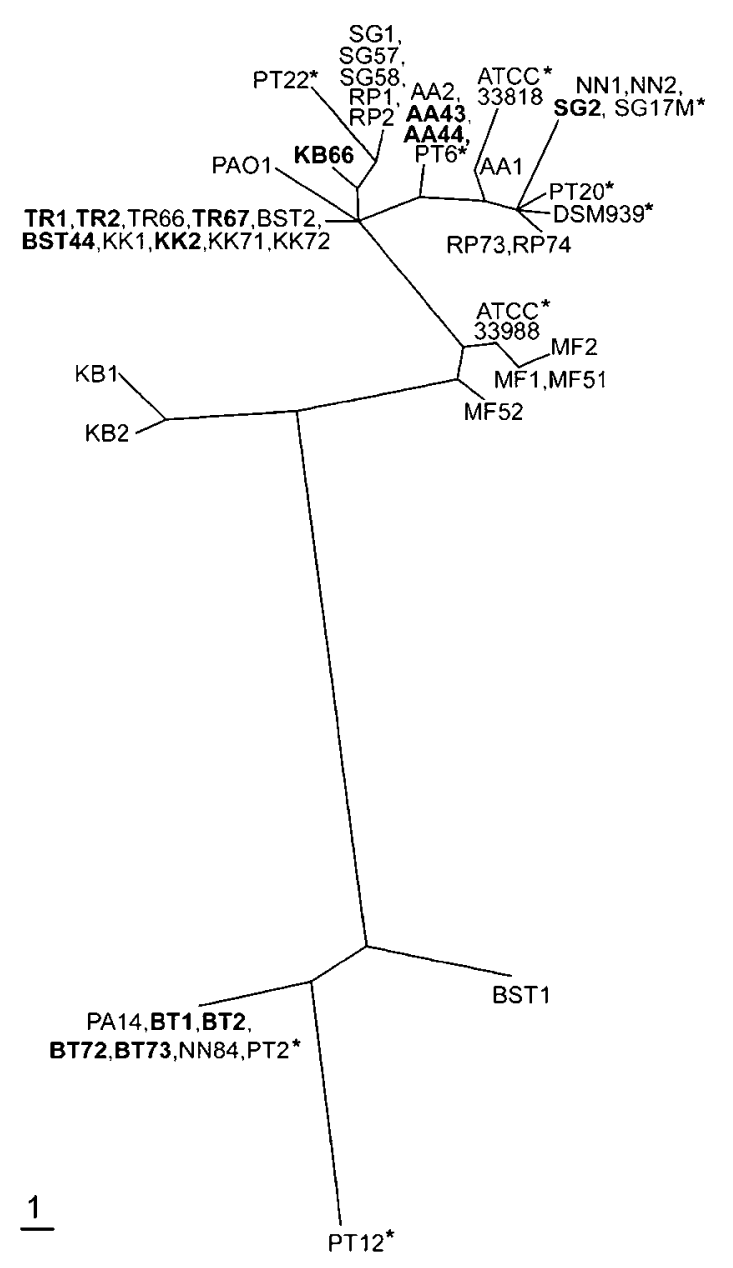

Fig. 2. Dendrogram based on mucABD genotypes of $37 \mathrm{CF}$ isolates and nine environmental isolates, with $P$. aeruginosa PAO1 and PA14 as reference strains. Strains carrying a mucA stop mutation are designated by bold type. Environmental strains are designated by an asterisk. Bar, 1 SNP.

recurrent emergence of the $\triangle \mathrm{G} 430$ mutation in independent CF lung habitats and unrelated mucABD genetic backgrounds (Table 2, Fig. 2) is in agreement with in vitro studies showing that this homopolymeric tract is prone to single nucleotide deletion upon exposure of $P$. aeruginosa biofilms to reactive oxygen species, a scenario envisaged to occur in the CF lung (Mathee et al., 1999).

Stop mutations in $m u c A$ are known to lead to alginate overproduction and conversion to a stable mucoid phenotype in P. aeruginosa (Martin et al., 1993b). We were able to confirm the causative role of the loss-offunction mutation in $m u c A$ for mucoidy in the reference strain PAO1 and its isogenic single mutant $\triangle \mathrm{G} 430 \mathrm{mucA}$ (footnote in Table 2). This association, however, was not so evident for the $\mathrm{CF}$ isolates. Just five of the 14 mucA stop mutants were overexpressing alginate (Table 2). During the chronic colonization of CF airways, $P$. aeruginosa is continuously accumulating mutations in its genome

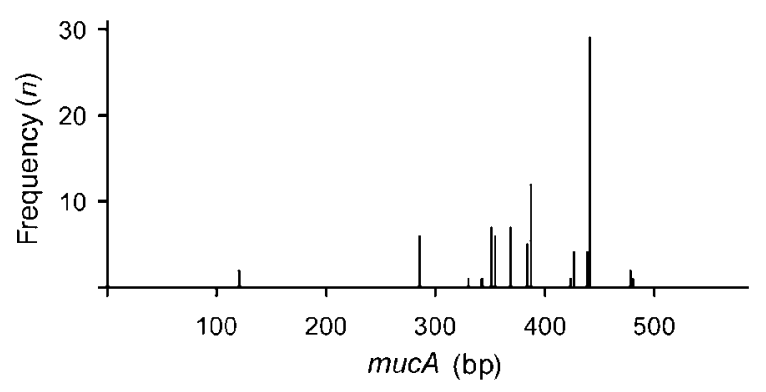

Fig. 3. Frequency and localization of stop mutations of the mucA gene of $P$. aeruginosa strains collected from the airways of individuals with CF in North America (Spencer et al., 2003; Yoon et al., 2006), Australia (Anthony et al., 2002) and Europe (this study).

(Smith et al., 2006); therefore, secondary site mutations probably caused the reversion to a non-mucoid morphotype in the majority of mucA mutant strains.

\section{DISCUSSION}

Chromosomal mosaic genes are a major source of genetic diversity, and are common in naturally transformable bacteria such as neisseriae and streptococci (Hakenbeck, 1998). In the non-transformable $P$. aeruginosa, mosaicism is known in the pyoverdine biosynthesis locus (Smith et al., 2006), ampC (PA4110) (Spangenberg et al., 1998), fleP (PA1096) (Arora et al., 2001), fliC (PA1092) (Spangenberg et al., 1998) and oprD (PA0958) (Pirnay et al., 2002b). This report adds the muc operon to the list of mosaic genes. Compared to the number of analysed clones and isolates, the frequency of intragenic recombination may appear to be high; however, this does not imply that this locus is subject to evolutionary forces additional to those of the bulk of the genome. Bacteria such as the ubiquitous and metabolically versatile $P$. aeruginosa are believed to have very large population sizes (Lynch \& Conery, 2003), and correspondingly are expected to evolve under purifying or negative natural selection, i.e. natural selection acting to decrease the frequency of deleterious alleles. The quantitative criterion is a low ratio of non-synonymous $\left(d_{\mathrm{N}}\right)$ over synonymous $\left(d_{\mathrm{S}}\right)$ nucleotide substitutions, and indeed, low $d_{\mathrm{N}} / d_{\mathrm{S}}$ ratios of $0 \cdot 14$ are observed when large datasets of phylogenetically independent pairs of genes are compared between closely related pairs of bacterial species (Friedman et al., 2004; Jordan et al., 2002). A significant excess of $d_{\mathrm{N}}$ over $d_{\mathrm{S}}$ nucleotide substitutions is interpreted as evidence for the action of positive selection. The $d_{\mathrm{N}} / d_{\mathrm{S}}$ ratio of 0.27 for the $m u c$ locus, however, is not significantly different from the global $d_{\mathrm{N}} / d_{\mathrm{S}}$ ratio of $\sim 0 \cdot 16$ for the $P$. aeruginosa core genome (Spencer et al., 2003). Hence, we conclude that the muc locus, like the core genome, is subject to ongoing purifying selection. 
Although at first glance the observed intragenic recombination rate seems to be high, it is in complete accordance with earlier reports that recombination is frequent in $P$. aeruginosa, and that correspondingly the population has an epidemic non-clonal population structure (Kiewitz \& Tümmler, 2000; Pirnay et al., 2002a). All genotypes, each of which is characterized by non-random association of alleles, are in linkage equilibrium to each other. In other words, the frequency of recombination is high enough to prevent linkage disequilibrium among genes and hitchhiking effects on the phenotype. Previous publications on the population genetics of $P$. aeruginosa have focused on the diversity of unrelated clones. By sequencing several strains of the same clone, we now demonstrate that interclonal recombination contributes to intraclonal variation and the generation of mosaic genes. The colonized CF lung is characterized by high numbers of $P$. aeruginos $a$ and only a few other bacterial competitors (Breitenstein et al., 1997). Such a scenario may facilitate the exchange of homologous genes between two or more $P$. aeruginosa clones.

Sequential isolates from CF lungs have been extensively characterized in one index case who became chronically colonized with $P$. aeruginosa very early, by the age of 6 months (Smith et al., 2006). Complete genome sequencing of clonally related month 6 and month 96 isolates uncovered the fixation of 68 mutations in the chromosome of the late isolate. No stop mutation in the mucA gene was observed in the month 96 isolate. A previous analysis on 36 sequential isolates recovered from the same patient during the first 30 months of airway colonization, however, identified eight mucoid strains, six of which carried either the $\Delta \mathrm{G} 357$ or the $\Delta \mathrm{G} 430$ stop mutation in mucA (Spencer et al., 2003). Lineages harbouring mucA mutations have not been detected in month 60 and month 96 isolates (see Fig. 1 in Smith et al., 2006). The disappearance of mucA mutants from the lungs of this early colonized patient is in accordance with our observation in the older patients 2 and 9 of our cohort (Table 2), who also lost mucA mutants during colonization. Our study on initial and late isolates from 10 patients moreover demonstrates that loss-of-function mutations in mucA either emerge de novo in the CF lung (see Table 2, patients 2, 3, 5, 6 and 10) or are acquired by nosocomial transmission (patients 4, 7 and 9).

In summary, the spectrum of loss-of-function mutations in the mucA gene of CF isolates is dominated by the major mutation $\Delta \mathrm{G} 430$ and a cluster of further stop mutations within $100 \mathrm{bp}$ upstream of $\Delta \mathrm{G} 430$. The dominant mutations are recurrent mutations on different mucABD haplotypes. No mutations were detected in the negative regulator $m u c B$ or in $m u c D$, which plays a dual role in the regulation of alginate production, as well as in temperature resistance (Wood \& Ohman, 2006). Sequencing of sequential isolates moreover provided direct experimental evidence for intragenic recombination as a means of generating intraclonal diversity in $P$. aeruginosa.

\section{ACKNOWLEDGEMENTS}

The study was supported in part by a Marie Curie Fellowship to A. B. (MCFI-2001-51061), and by the Mukoviszidose eV, Bonn, Germany, and the Italian Cystic Fibrosis Foundation. J. K. and N. C. are members of the Deutsche Forschungsgemeinschaft (DFG)-sponsored Research Training Group 'Pseudomonas: Pathogenicity and Biotechnology'.

\section{REFERENCES}

Anthony, M., Rose, B., Pegler, M. B. \& 7 other authors (2002). Genetic analysis of Pseudomonas aeruginosa isolates from the sputa of Australian adult cystic fibrosis patients. J Clin Microbiol 40, 2772-2778.

Arora, S., Bangera, S., Lory, S. \& Ramphal, R. (2001). A genomic island in Pseudomonas aeruginosa carries the determinants of flagellin glycosylation. Proc Natl Acad Sci U S A 98, 9342-9347.

Breitenstein, S., Walter, S., Bosshammer, J., Römling, U. \& Tümmler, B. (1997). Direct sputum analysis of Pseudomonas aeruginosa macrorestriction fragment genotypes in patients with cystic fibrosis. Med Microbiol Immunol (Berl) 186, 93-99.

Curran, B., Jonas, D., Grundmann, H., Pitt, T. \& Dowson, C. G. (2004). Development of a multilocus sequence typing scheme for the opportunistic pathogen Pseudomonas aeruginosa. J Clin Microbiol 42, 5644-5649.

Dayhoff, M. O., Schwartz, R. M. \& Orcutt, B. C. (1978). A model of evolutionary change in proteins. In Atlas of Protein Sequence and Structure, vol. 5, suppl. 3, pp. 345-352. Edited by M. O. Dayhoff. Washington, DC: National Biomedical Research Foundation.

Dinesh, S. D., Grundmann, H., Pitt, T. L. \& Römling, U. (2003). European-wide distribution of Pseudomonas aeruginosa clone C. Clin Microbiol Infect 9, 1228-1233.

Ernst, R. K., D’Argenio, D. A., Ichikawa, J. K. \& 12 other authors (2003). Genome mosaicism is conserved but not unique in Pseudomonas aeruginosa isolates from the airways of young children with cystic fibrosis. Environ Microbiol 5, 1341-1349.

Felsenstein, J. (2002). PHYLIP (Phylogeny Inference Package) version 3.6 (alpha 3). Distributed by the author. Seattle, WA: University of Washington.

Feltman, H., Schulert, G., Khan, S., Jain, M., Peterson, L. \& Hauser, A. R. (2001). Prevalence of type III secretion genes in clinical and environmental isolates of Pseudomonas aeruginosa. Microbiology 147, 2659-2669.

French, S. \& Robson, B. (1983). What is a conservative substitution? J Mol Evol 19, 171-175.

Friedman, R., Drake, W. J. \& Hughes, A. L. (2004). Genome-wide patterns of nucleotide substitution reveal stringent functional constraints on the protein sequences of thermophiles. Genetics 167, 1507-1512.

Hakenbeck, R. (1998). Mosaic genes and their role in penicillinresistant Streptococcus pneumoniae. Electrophoresis 19, 597-601.

Jordan, I. K., Rogozin, I. B., Wolf, V. I. \& Koonin, E. V. (2002). Microevolutionary genomics of bacteria. Theor Popul Biol 61, 435-447.

Kiewitz, C. \& Tümmler, B. (2000). Sequence diversity of Pseudomonas aeruginosa: impact on population structure and genome evolution. J Bacteriol 182, 3125-3135.

Kresse, A. U., Dinesh, S. D., Larbig, K. \& Römling, U. (2003). Impact of large chromosomal inversions on the adaptation and evolution of Pseudomonas aeruginosa chronically colonizing cystic fibrosis lungs. Mol Microbiol 47, 145-158. 
Lam, J., Chan, R., Lam, K. \& Costerton, J. W. (1980). Production of mucoid microcolonies by Pseudomonas aeruginosa within infected lungs in cystic fibrosis. Infect Immun 28, 546-556.

Larbig, K. D., Christmann, A., Johann, A., Klockgether, J., Hartsch, T., Merkl, R., Wiehlmann, L., Fritz, H. J. \& Tümmler, B. (2002). Gene islands integrated into tRNA(Gly) genes confer genome diversity on a Pseudomonas aeruginosa clone. J Bacteriol 184, 6665-6680.

Liberati, N. T., Urbach, J. M., Miyata, S., Lee, D. G., Drenkard, E., Wu, G., Villanueva, J., Wie, T. \& Ausubel, F. M. (2006). An ordered, nonredundant library of Pseudomonas aeruginosa strain PA14 transposon insertion mutants. Proc Natl Acad Sci U S A 103, 2833-2838.

Lynch, M. \& Conery, J. S. (2003). The origin of genome complexity. Science 302, 1401-1404.

Martin, D. W., Schurr, M. J., Mudd, M. H. \& Deretic, V. (1993a). Differentiation of Pseudomonas aeruginosa into the alginate-producing form: inactivation of $m u c B$ causes conversion to mucoidy. $\mathrm{Mol}$ Microbiol 9, 497-506.

Martin, D. W., Schurr, M. J., Mudd, M. H., Govan, J. R. W., Holloway, B. W. \& Deretic, V. (1993b). Mechanisms of conversion to mucoidy in Pseudomonas aeruginosa infecting cystic fibrosis patients. Proc Natl Acad Sci U S A 90, 8377-8381.

Mathee, K., McPherson, C. J. \& Ohman, D. E. (1997). Posttranslational control of the $\operatorname{alg} T(\operatorname{alg} U)$-encoded $\sigma^{22}$ for expression of the alginate regulon in Pseudomonas aeruginosa and localization of its antagonist proteins MucA and MucB (AlgN). J Bacteriol 179, 3711-3720.

Mathee, K., Ciofu, O., Sternberg, C. \& 9 other authors (1999). Mucoid conversion of Pseudomonas aeruginosa by hydrogen peroxide: a mechanism for virulence activation in the cystic fibrosis lung. Microbiology 145, 1349-1357.

Morales, G., Wiehlmann, L., Gudowius, P., van Delden, C., Tümmler, B., Martinez, J. L. \& Rojo, F. (2004). Structure of Pseudomonas aeruginosa populations analyzed by single nucleotide polymorphism and pulsed-field gel electrophoresis genotyping. J Bacteriol 186, $4228-4237$.

Pirnay, J. P., De Vos, D., Cochez, C., Bilocq, F., Vanderkelen, A., Zizi, M., Ghysels, B. \& Cornelis, P. (2002a). Pseudomonas aeruginosa displays an epidemic population structure. Environ Microbiol 4, 898-911.

Pirnay, J. P., De Vos, D., Mossialos, D., Vanderkelen, A., Cornelis, P. \& Zizi, M. (2002b). Analysis of the Pseudomonas aeruginosa oprD gene from clinical and environmental isolates. Environ Microbiol 4, 872-882.

Ramsey, D. M. \& Wozniak, D. J. (2005). Understanding the control of Pseudomonas aeruginosa alginate synthesis and the prospects for management of chronic infections in cystic fibrosis. Mol Microbiol 56, 309-322.

Römling, U., Fiedler, B., Bosshammer, J., Grothues, D., Greipel, J., von der Hardt, H. \& Tümmler, B. (1994). Epidemiology of chronic Pseudomonas aeruginosa infections in cystic fibrosis. J Infect Dis 170, 1616-1621.

Schurr, M. J., Yu, H., Martinez-Salazar, J. M., Boucher, J. C. \& Deretic, V. (1996). Control of AlgU, a member of the $\sigma^{\mathrm{E}}$-like family of stress sigma factors, by the negative regulators MucA and MucB and Pseudomonas aeruginosa conversion to mucoidy in cystic fibrosis. J Bacteriol 178, 4997-5004.

Smith, E. E., Buckley, D. G., Wu, Z. \& 10 other authors (2006). Genetic adaptation by Pseudomonas aeruginosa to the airways of cystic fibrosis patients. Proc Natl Acad Sci U S A 103, 8487-8492.

Spangenberg, C., Heuer, T., Bürger, C. \& Tümmler, B. (1996). Genetic diversity of flagellins of Pseudomonas aeruginosa. FEBS Lett 396, 213-217.

Spangenberg, C., Montie, T. C. \& Tümmler, B. (1998). Structural and functional implications of sequence diversity of Pseudomonas aeruginosa genes oriC, ampC and fliC. Electrophoresis 19, 545-550.

Spencer, D. H., Kas, A., Smith, E. E., Raymond, C. K., Sims, E. H., Hastings, M., Burns, J. L., Kaul, R. \& Olson, M. V. (2003). Wholegenome sequence variation among multiple isolates of Pseudomonas aeruginosa. J Bacteriol 185, 1316-1325.

Taylor, W. R. (1986). The classification of amino acid conservation. $J$ Theor Biol 119, 205-218.

Tümmler, B. (2006). Clonal variations in Pseudomonas aeruginosa. In Pseudomonas, vol. 4, pp. 35-68. Edited by J.-L. Ramos \& R. C. Levesque. Heidelberg: Springer.

Wood, L. F. \& Ohman, D. E. (2006). Independent regulation of MucD, an HtrA-like protease in Pseudomonas aeruginosa, and the role of its proteolytic motif in alginate gene regulation. J Bacteriol 188, 3134-3137.

Yoon, S. S., Coakley, R., Lau, G. W. \& 12 other authors (2006). Anaerobic killing of mucoid Pseudomonas aeruginosa by acidified nitrite derivatives under cystic fibrosis airway conditions. J Clin Invest 116, 436-446. 